Spektroskopisch ähnliche Verhältnisse treten auch bei Anwesenheit zweier benachbarter Carbonylgruppen im Molekiil des absorbierenden Stoffes auf. Wie bei den Keto- Enoltautomeren wird auch durch diese Atomgruppierung nach A. W. Stewart und E. C. C. $B a\left(y^{66}\right)$ ein Oscillationszustand bedingt, der aber im Gegensatz zu dem zuerst besprochenen die Absorption von mit größeren. Wellenlängen zur Folge hat (d.h. im Blau), so dab diese Stotfe gelb 'erscheinen. Beim Diacetyl geben die Verff. die Oscillationen dureh folgendes Sehema wieder :

$$
\begin{array}{lll}
\mathrm{CH}_{3}-\mathrm{C}=\mathrm{O} & \longrightarrow \\
\mathrm{CH}_{3}-\mathrm{C}=\mathrm{O} & \longrightarrow \mathrm{CH}_{3}-\mathrm{C}-\mathrm{O} \\
\mathrm{CH}_{3}-\mathrm{C}-\mathrm{O}
\end{array}
$$

und nemmen den die Absorption bedingenden Vorgang Is orropesis das charakteristische $A b$ sorptionsband ein is or r o p is ches.

Ganz analoge Erscheinungen sind bei Chinonen beobachtet. Benzochinon hat ein isorropisches Band und zeigt keine Anzeichen von benzolischer Struktur. Die Rinführung von Methylgruppen sowie von Chlor vermindert die Beständigkeit des isorropischen Bandes und ruft ein benzolisches Band im Spectrum hervor; auch die chemische Reaktionsfähigkeit der Carbonylgruppen scheint mit dem isorropischen Vorgang in Beziehung zu stehen.

Von diesen neuen Methoden der spektroskopischen Konstitutionsbestimmungen sind sicher sowohl für Keto-Enoltautomere als für Chinoide wichtige Einblicke in feinere Isomerieerscheinungen zu erwarten und man muß deshalb den weiteren Arbeiten Balys mit Spannung entgegensehen.

\section{Über die Analysen einiger römischer Gläser und Bronzen.}

\section{Von F. Henrich und P. Roters.}

\section{(Eingeg. d. 21.6. 1907.)}

Vor einiger Zeit erhielt der eine von uns von Herrn Baurat H. J a c o b j jun. in Homburg v. d.H. ein Stückchen intensiv gefärbtes Glas, das bei den Ausgrabungen auf dem Römerkastell Saalburg bei Homburg zutage getreten und sicher römischen Ursprungs war. Eine qualitative Analyse ergab, daß ein Natronglas vorlag, das als Nebenbestandteile Eisen und Mangan enthielt. Um über die Beziehungen beider zur Farbe des Glases etwas zu erfahren, unterwarfen wir eine Anzahl von Glasscherben sicher römischen Ursprungs einer genauen quantitativen Analyse.

Die weitaus meisten römischen Gläser, die man in Nuseen und Sammlungen sieht, sind mehr oder weniger grün gefärbt, und viele zeigen auf ihrer Oberfläche perlmutterartigen Glanz. Die Ansicht, daB den Römern die Herstellung farbloser Gläser unbekannt gewesen sei, ist längst durch Funde bei Ausgrabungen widerlegt. Das antike Glas solite als Rival der Edelsteine bunt sein, so verlangte es lange Zeit die Wode, und erst seit Ende des ersten Jahrhunderts nach Chr. werden die farblosen Gläser

65) Proc. Chem. Soc. 22, 34, 35.

populärer. Bei den Ausgrabungen auf der Saalburg wurde denn auch eine große Anzahl Glasscherben römischen Ursprungs zutage gebracht, die alle Ubergänge von intensiv dunkelgrün gefärbten zu fast farblosen Gläsern darstellen. Von einer Anzah] solcher Scherben unzweifelhaft römischen Ursprungs, die Herr $J$ a c o b i jr. uns gütigst zur Verfügnug stellte, wählten wir vierStücke von ungefähr gleicher Dicke aus. Das cine (I) hatte eine tiof dunkelgrüne, satte Farbe, während ein anderes (IV) in der Durchsicht völlig farblos erschien und nur auf den Bruch. flächen einen schwach grünen Schimmer erkennen lie 3. Von den beiden anderen war das eine (III) in der Durchsicht schwach grün gefärbt, das andere (II) zeigte ein stärkeres Grün, aber nicht von der Intensität wie das zuerst erwähnte. In diesen vier Proben waren die wichtigsten Phasen eines allmählichen Ubergangs von farblos bis zum intensivsten noch durchsichtigen Grün vertreten.

Die Analysen ergaben folgende Resultate:

$$
\begin{aligned}
\text { tief dunkelgrünes Glas (I) } \\
\mathrm{SiO}_{2}=66,54 \% \\
\mathrm{Al}_{2} \mathrm{O}_{3}=1,95 \% \\
\mathrm{Fe}_{2} \mathrm{O}_{3}=1,74 \% \\
\mathrm{CaO}=6,07 \\
\mathrm{MgO}=038 \% \\
\mathrm{Na}_{2} \mathrm{O}=23,73 \\
100,41 \%
\end{aligned}
$$

schwachgr ünes Glas (III)

$$
\begin{aligned}
& \mathrm{SiO}_{2}=68,54 \% \\
& \mathrm{Al}_{2} \mathrm{O}_{3}=1,85 \% \\
& \mathrm{Fe}_{2} \mathrm{O}_{3}=0,48 \% \\
& \mathrm{CaO}=6,45 \% \\
& \mathrm{MgO}=0,65 \% \\
& \mathrm{Na}_{2} \mathrm{O}=21,61 \\
& -\frac{99,58}{2}
\end{aligned}
$$$$
\text { grünes Glas (II) }
$$$$
\mathrm{SiO}_{2}=69,44 \%
$$$$
\mathrm{AI}_{2} \mathrm{O}_{3}=3,79 \%
$$$$
\mathrm{Fc}_{2} \mathrm{O}_{3}=0,82 \%
$$$$
\mathrm{CaO}=6.53 \%
$$$$
\mathrm{MgO}=0,63 \%
$$$$
\mathrm{Na}_{2} \mathrm{O}=\frac{18,71 \%}{99,92}
$$

farbloses Glas (TV) $\mathrm{SiO}_{2}=67,00 \%$ $\mathrm{Al}_{2} \mathrm{O}_{3}=4,21 \%$ $\mathrm{Fe}_{2} \mathrm{O}_{3}=0,49 \%$ $\mathrm{CaO}=6,75 \%$ $\mathrm{MgO}=0,54 \%$ $\mathrm{MnO}=0,36 \%$ $\mathrm{Na}_{2} \mathrm{O}=20,67 \%$ 100,02

Man sieht, daß überall Natrongläser vorlagen, und daß von den Ingredienzien, die die Farbe bedingen, der Eisengehalt bei den ersten drei Gläsern mit der Intensität der grünen Färbung abnimmt.

Um dies Resultat zu kontrollieren, suchte ich an den zur Verfügung stehonden Scherben zwei weitere Stichproben aus, ein schwach und ein etwas stärker hellgrün gefärbtes Glas. Die Analysen ergaben folgendes Resultat:

schwach hellgrïn
gefürbtes Glas $(\mathrm{Y})$
$\mathrm{SiO}_{2}=67,78 \%$
$\mathrm{Al}_{2} \mathrm{O}_{3}=2,12 \%$
$\mathrm{Fe}_{2} \mathrm{O}_{3}=0,48 \%$
$\mathrm{CaO}=6,74 \%$
$\begin{aligned} \mathrm{MgO} & =0,64 \% \\ \mathrm{Na} & =21,72 \% \\ & =99,48\end{aligned}$

$$
\begin{gathered}
\text { etwas stärker hellgrim } \\
\text { gefärtotes } \mathrm{Glas}(\mathrm{IV}) \\
\mathrm{SiO}_{2}=67,55 \% \\
\mathrm{Al}_{2} \mathrm{O}_{3}=4,08 \% \\
\mathrm{Fe}_{2} \mathrm{O}_{3}=0,59 \% \\
\mathrm{CaO}=6,74 \% \\
\mathrm{MgO}=0,54 \% \\
\mathrm{Na} \mathrm{O}=20,67 \% \\
=100,17 \%
\end{gathered}
$$

also eine Bestätigung des oben Gefundenen.

Damit ist auch experimentell bewiesen, was von vornherein zu erwarten war, daß nämlich die grüne Farbe der römischen Gläser in der Hauptsache durch kieselsaures Eisenoxydul bedingt wird.

Aber noch in einer andern Hinsicht sind die Analysen lehrreich. In allen Glasscherben konnte man neben Eisen auch Mangan nachweisen. Als aber von jeder Probe je ein Gramm der quantitativen Analyse unterworfen wurde, war da das Mangan in 
fünf von sechs Fällen quantitativ nicht bestimmbar und nur in einer - der farblosen Probe (IV) - in solcher Menge vorhanden, daß es prozentual ausgedrückt werden konnte. Diese Tatsachen weisen zunächst darauf hin, daß man schon damals Manganoxyde als Entfärbungsmittel für Glas anwandte, wie uns das auch überliefert ist ${ }^{1}$ ).

Vergleichen wir aber die Analysen der schwach grünen Gläser II und V mit der des farblosen Glases IV, so sehen wir, daB der Eisengehalt aller ziemlich gleich ist, ja beim farblosen Glas noch etwas mehr beträgt, als bei den gefärbten. Nun wurde aber schon durch L i e b i g erwiesen, daßs die Farblosigkeit des Glases nicht etwa dadurch hervorgerufen wird, daß das Mangansuperoxyd das stark grün gefärbte Eisenoyxdulsilicat zum schwächer gelb gefärbten Eisenoxydsilicat oxydiert. Vielmehr ist die Farbe des Mangansilicats komplementär zu der des grünen Eisenoxydulsilicats. Dadurch heben sich bei richtiger Mischung beide Farben auf, und es entsteht ein farbloses Glas. So erklärt es sich, daß das Glas Nr. IV im Gegensatz zu IIT und V bei ungefähr gleichem Eisengehalt farblos erscheint.

Unter den erhaltenen Glasscherben befand sich einer, der von einer dicken grünlichbraunen Kruste umkleidet war. Beim Abkratzen derselben hinterblieb ein grüner Glaskern, der etwas trübe erschien und nun jenen perlmutterartigen Glanz zeigte, den man öfters an antiken Gläsern bemerkt. Das Aussehen der Kruste unterschied sich von der Erde, aus der dieScherben ausgegraben waren, sehr wesentlich, und der Augenschein machte es vor vornherein wahrscheinlich, daß die Kruste ein Zersetzungsprodukt des Glases ist. In der 'Tat fanden sich bei der Analyse alle Bestandteile des Glases auch in der Kruste. Nimmt man an, daß die Kruste wirklich durch Zersetzung des Glases erzeugt wurde, so ist es einleuchtend, daß diese Zersetzung obwohl,

1) Vergl. Römische Altertümer der Sammlung C. A. Nissen von Dr. Kisa S. 6. die ganze Oberfläche des Glases ergreifend nicht so gleichmäßig nach innen erfolgen kann, daß genau parallele Schichten abgelöst werden. Viel wahrscheinlicher ist es, daß eine unregelmäBige Oberfläche am unzersetzten Glase entsteht, die, wenn das Glas zugleich trübe geworden ist, das Licht an der Oberfläche so reflektiert, daß jener perlmutterartige Glanz erscheint.

Auch eine Anzahl von Bronzeproben hatte uns ILerr $\mathrm{J}$ a c o b i jun. gütigst zur Verfügung gestellt. Eine römische, silberfarbige war von besonderem Interesse, da sie bei den Ausgrabungen ofters zutage trat und gut erhalten war. Sie enthielt Kupfer, Blei und Zinn in folgenden Mengenverhältnissen :

$$
\begin{array}{cr}
\text { I } & \text { II } \\
\mathrm{Cu}=73,96 \% & 73,65 \% \\
\mathrm{~Pb}=24,17 \% & 24,16 \% \\
\mathrm{Sn}=2,37 \% & 2,37 \% \\
100,50 & 100,18
\end{array}
$$

Eine dunklere römisu'k Bronze zeigte folgende Zusammensetzung :

$$
\begin{array}{cr}
\mathrm{I} & \multicolumn{1}{c}{\text { II }} \\
\mathrm{Cu}=84,87 \% & 85,16 \% \\
\mathrm{~Pb}=13,82 \% & 13,52 \% \\
\mathrm{Sn}=1,28 \% & 1,11 \%
\end{array}
$$

Endlich untersuchten wir noch cin als, prähistorische" Bronze bezeichnetes Stick. Es war ein Teil eines großen Ringes und bestand aus einem bronzefarbigen Kern, der die Metallegierung noch unverändert enthielt, und aus einer dicken Patinakruste. Beide wurden nach Möglichkeit voneinander getrennt und analysiert. Leider war das Material bei der Patina beschränkt, so daß nicht alle Bestandteile quantitativ bestimmt werden konnten:

$\begin{array}{cc}\text { a) Kern } & \text { b) Patina } \\ \mathrm{Cu}=97,09 \% & \mathrm{Cu}=32,64 \% \\ \mathrm{Sn}=2,40 \% & \mathrm{Sn}=2,39 \% \\ \mathrm{~Pb}=0,29 \% & \mathrm{~Pb}=0,27 \% \\ & \mathrm{CO}_{2}=6,66 \%\end{array}$

\section{Referate.}

\section{8. Elektrochemie.}

Francis B. Crocker. Die Deckersche Primärzelle. (Transact. Amer. Electrochemical Society, New York. 8./9. Okt. 1906; nach Electrochemical and Metallurgical Industry 4, 441.) Das von F. A. D e c k e r in Philadelphia hergestellte Element enthält Zinkplatten in verd. Schwefelsäure und Graphitplatten in einer Lösung von Natriumbichromat und Schwefelsäure. Je eine Zinkplatte befindet sich, zusammen mit der Schwefelsäure, in einem flachen, porösen TongefäB. Diese Gefäße werden in der Weise hergestellt, da 3 zwei unglasierte irdene Platten mit verdickten Rändern und Querrippen in Stahlformen, jede fï̈r sich, geformt und darauf miteinander vereinigt werden. Die Wände werden sodann so dünn geschliffen, daß das Licht hindurchscheinen kann. Um die gewünschte Porosität zu erhalten, wird eine besondere Tonmischung dazu verwendet. Die Graphitplatten werden direkt gegen die Außenwände der porösen Gefäße gestellt. Infolge der Dünnheit der Gefäßwände und der Nähe der Zink- und Graphitplatten zueinander ist der innere Widerstand der Zelle nur gering. Mehrere solcher Zellen bilden eine Batterie, die in einen Behälter aus Hartgummi eingesetzt wird. Die von dem Verf. mit 1,9-1,3 Volt ausgeführton Versuche haben eine Ausbeute von 14,7 Wattstunden fiir 1 Pfd. des Gesamtgewichtes ergeben. Wine Zelle ron $17 \mathrm{Pfd}$. Gewicht liefert, bei durchschnittlich ungefähr 1,7 Volt, 150 Ampèrestunden oder ungefähr 250 Wattstunden, was $1 / 3$ PS.-Stunde gleichkommt. Die für die Erzeugung von 1. PS.-Stunde erforderlichen Mengen Zink, Schwefelsäure und Natriumbichromat kosten, wenn die Stoffe nach einmaligem Gebrauch fortgeworfen werden, ungefähr 35 Cents. Man darf daher erwarten, daB die $\mathrm{D}$ e c k c r sche Zelle weg? ihrer Billigkeit eine ausgedehnte Anwendung, namentlich für die Beleuchtung von Eisenbahnen, finden wird. 